\title{
VORWORT UND DANK
}

Dieser Band ist das Ergebnis einer am 11. und 12. Januar 2010 in Paris unter dem Titel »Faire la paix et se défendre à la fin du Moyen Âge/Frieden schaffen und sich verteidigen im Spätmittelalter« von der Fondation Maison des sciences de l'homme (FMSH, Paris) in Zusammenarbeit mit dem Deutschen Historischen Institut Paris (DHIP) und der Unterstützung des Deutschen Akademischen Austauschdienstes (DAAD) durchgeführten internationalen Tagung. An der Veranstaltung, die im DHIP und der FMSH stattfand, nahmen Wissenschaftler aus Frankreich, Deutschland, Spanien und Belgien teil. Der deutsch-französische Dialog stand im Vordergrund. Als wissenschaftliche Organisatorin danke ich den genannten Institutionen herzlich für ihre Unterstützung. Für diese Phase gilt mein Dank besonders der FMSH und ihren Leitern, Alain d'Iribarne, directeur de recherche (CNRS) und Michel Wieviorka, directeur d'études (EHESS), die es mir ermöglicht haben, das Projekt auf den Weg zu bringen. Für ihre engagierte Begleitung danke ich Hinnerk Bruhns, directeur de recherche émérite (CNRS) und seinem Nachfolger im deutsch-französischen Programm der FMSH, Falk Bretschneider, maître de conférences (EHESS). Auf Seiten des DHIP und des DAAD gilt mein Dank Prof. Dr. Gudrun Gersmann, Prof. Dr. Rolf Große und Dr. Klaudia Knabel. Als Sektions- und Diskussionsleiter haben Claude Gauvard, professeur émérite (Univ. Paris I), Prof. Dr. Rolf Große, Prof. Dr. Robert Jacob (Univ. de Liège) und Prof. Dr. Pierre Monnet, Direktor des Institut français d'histoire en Allemagne sehr interessante und anregende Beiträge geleistet. Bei der praktischen Organisation waren mir Raquel Sanz-Barrio (FMSH), Margarete Martaguet und Dunja Houelleu (DHIP) eine große Hilfe.

Frau Prof. Dr. Gudrun Gersmann, Direktorin des DHIP, danke ich ganz herzlich für die Aufnahme des Bandes in die Pariser Historischen Studien. Die Publikation wurde vom französischen Verteidigungsministerium (Ministère de la Défense et des Anciens Combattants, direction de la Mémoire, du Patrimoine et des Archives) unterstützt. Ich möchte mich an dieser Stelle für das freundliche Interesse bedanken, das dort Laurent Veyssière, chef de la délégation des Patrimoines culturels und Anne Heuzé von Anfang an dem Vorhaben entgegengebracht haben. Mein herzlicher Dank gilt auch Françoise Hildesheimer, conservateur général (Archives nationales). Veronika Vollmer (DHIP) hat die Druckvorbereitung des Manuskripts stets sehr engagiert und sorgfältig betreut. Im DHIP waren Ralf Nädele und Myriam Olivier ebenfalls an der praktischen Umsetzung des Projektes beteiligt.

Paris, 10. Oktober 2011

Gisela Naegle 